\title{
Use of Solar Panels in the Design of Small CubeSat Spacecraft
}

\author{
Kirill V. Selivanov ${ }^{1,}{ }^{*}$, Igor A. Vasiliev ${ }^{2}$, and Arina A. Yakovenko ${ }^{1}$ \\ ${ }^{1}$ Department of Design and Technology of Electronic Devices, Bauman Moscow State Technical \\ University, 5, 2-aj Baumanskaya str., Moscow, Russian Federation \\ ${ }^{2}$ Department of electrical engineering and industrial electronics, Bauman Moscow State Technical \\ University, 5, 2-aj Baumanskaya str., Moscow, Russian Federation
}

\begin{abstract}
The article discusses organization of power supply for small spacecraft of CubeSat type. Various methods of power supply of CubeSat onboard equipment are worked out. The use of solar panels (SP) for power supply of CubeSat is proposed. The developed technology for the production of solar arrays of the required size from solar panels has been demonstrated. The organization of incoming control of solar panels to improve reliability and achieve maximum efficiency of produced solar panels is considered. A developed stand for diagnostics of solar cells, which allows detecting latent defects, is presented. The issue of determining power characteristics of the developed solar panels and the necessary optimal load for their maximum efficiency is discussed. The installation of solar panels on the CubeSat aircraft is described. The efficiency of solar panels is tested in space by launching CubeSat on a stratospheric probe. The parameters of the aircraft current supply and current generation by solar panels obtained as a result of the experiment are collected and processed, the results are presented in the article in the form of graphs. On basis of the data obtained, effectiveness of the use of solar panels on small aircraft of the CubeSat type is shown.
\end{abstract}

\section{Introduction}

Since the discovery of photoelectric effect and practical proof of the possibility of obtaining electricity by converting the energy of light in the mid-1950s, the development of photovoltaics hasn't stopped [1]. At the moment, the development of solar energy as part of the renewable energy sources (RES) industry is widely used and developed with high intensity. The main source of light on our planet is the sun. Sunlight can be considered one of the primary energy sources. Other types of primary energy include the energy released during the birth and formation of our planet. However, the supply of this type of energy, represented by volcanic eruptions and earthquakes, at the present stage of science and technology development cannot be used for the needs of mankind. The energy resources of our planet, such as gas, oil, coal, wind energy, hydropower and others, are secondary derivatives of solar energy. This fact gives rise to the assumption that solar energy in the

\footnotetext{
*Corresponding author: aselivanov_kv@mail.ru
} 
future, at one of the stages of its development, will be able to supply the maximum amount of energy for mankind and surpass all currently available energy sources [2,3,4].

The energy of sunlight is used to generate heat through the use of solar collectors and to generate electricity through the use of a large number of solar panels (SP), collected into solar arrays (SA). In terms of the volume of power generation under normal conditions, SAs are currently significantly inferior to more developed methods of power generation from fossil hydrocarbon energy sources, such as coal, oil, gas and others. SAs already occupy leading positions in specific areas $[5,6]$.

\section{Literature review}

Thus, solar arrays have a predominant share in generation of electricity for the space industry. The main objects for the supply of electricity generated by solar arrays include space stations, satellites, lunar rovers, mars rovers and other planetary rovers, as well as probes and other spacecraft. The specificity of use of this technique makes it impossible to supply it with other methods of power generation, except for the generation of electricity by solar arrays $[7,8]$. This is due to the following positive properties of SA power generation:

- $\quad$ No need for fuel delivery.

- Widespread availability of sunlight.

- $\quad$ Ease of transition of SAs between operation modes.

- DC generation.

- Good weight/power ratio.

- Ability to control the power received when creating a solar array.

The actual application and organization of SA power generation, especially in the space industry, is complicated by a number of factors and specific requirements that are present when using SA. This article is devoted to the analysis of the specifics of the use of SA for spacecraft, the development of electrical solutions and verification of their effectiveness in the practical launch of a stratospheric vehicle with a SA on board.

The spacecraft to be launched was designed in the CubeSat $2 U$ format. CubeSat is a program for creation of small and ultra-small artificial earth satellites. The miniaturization of electronic devices is a consequence of significant scientific progress in mechatronics and microelectronics, which has made it possible to significantly reduce the size of industrial solutions in the production of spacecraft. Thus, CubeSat assumes the principle of constructing products from modules measuring $10 \times 10 \times 10 \mathrm{~cm}$ and weighing no more than $1.33 \mathrm{~kg}$. The arrangement of several such modules into a single satellite is possible. However, each module is autonomous, and the output of one of the modules does not affect the operation of the others [8].

Initially, Li-ion rechargeable batteries were used to power the modules, which are still widely used in CubeSat products. However, during their operation, the following weaknesses of this solution were identified:

- $\quad$ Finite volume of the charge is limited.

- Risk of explosion of Li-ion battery at high temperatures.

- $\quad$ Li-ion battery failure at low temperatures.

- $\quad$ Significant weight of Li-ion batteries and related equipment.

Most of these shortcomings are caused or significantly aggravated by the specifics of Li-ion battery operation in the upper atmosphere and space. The temperature drop in the stratosphere from -60 to +20 degrees Celsius requires creation of a thermoregulation system for using $\mathrm{Li}$-ion rechargeable batteries in these conditions. In the presence of a large number of Li-ion rechargeable batteries, the thermal control system also becomes larger, requires more power and no longer fits into the specified parameters. To reduce detrimental properties of $\mathrm{Li}$-ion batteries as part of microsatellites, it was proposed to use solar panels 
(SP) in the form of solar arrays (SA) [8]. A part of Li-ion batteries was left as a source of backup power and buffer storage for the electricity generated by the solar array $[9,10,11]$.

To test the effectiveness of the proposed solution, an experiment was carried out to test the proposed concept in practice.

For this purpose, a spacecraft with 2 cubes of the CubeSat $2 \mathrm{U}$ design was developed and a stratospheric probe was developed to launch CubeSat $2 \mathrm{U}$ into the stratosphere. The stratosphere recreates as closely as possible the operating conditions in which space satellites operate, and using a probe to achieve it significantly reduces the cost of an experiment compared to using a satellite.

\section{Materials and methods}

The first stage of work on the spacecraft was its energy calculation. The main consumer of electricity on the probe is the thermal control system, which maintains the required temperature for probe operation and radio transmission system that provides communication with the ground. The rest of the probe systems - measurement sensors and microcontrollers - consume significantly less electricity, but it is their power supply that is a priority. All systems that consume electricity are presented in Table 1. It is worth explaining that in the name of CubeSat $2 U$ the index ' $2 U$ ' indicates that this device consists of 2 modules. The modules are designated U1 and U2 and, according to the development paradigm, each module is independent, can work autonomously and have individual equipment. Thus, the radio communication module with the ground is located only on U1.

Multiplying the power of all elements by their number, we get the power of each module, which will be $4.28 \mathrm{~W}^{*} \mathrm{~h}$ and $1.079 \mathrm{~W} * \mathrm{~h}$ for modules $\mathrm{U} 1$ and $\mathrm{U} 2$, respectively. The power of the heating equipment of the device is not considered, since it is individual for each device and depends on the specifics of operation and energy-saving technologies used in the product, and can also vary from units to tens of watts. In some cases, spacecraft can be turned off in a zone of low temperatures and re-turned on when entering the zone of acceptable operating temperatures.

The anticipated flight time of the probe on the stratospheric balloon will be about 2 hours, which means that the anticipated power consumption will be about $8.56 \mathrm{~W}^{*} \mathrm{~h}$ and $2.158 \mathrm{~W}^{*} \mathrm{~h}$ for modules $\mathrm{U} 1$ and $\mathrm{U} 2$, respectively.

To supply power and ensure maximum peak currents, $4 \mathrm{Li}$-ion batteries with a total capacity of $37 \mathrm{~W} / 1$ module in each CubeSat $2 \mathrm{U}$ module were used.

The question of overall efficiency of current generation by solar arrays on the stratospheric apparatus under the conditions we are considering remains open. Solar remediation in the stratosphere is about $1200-1400 \mathrm{~W} / \mathrm{m}^{2}$ of radiation power, which creates favorable conditions for maximum power generation by the solar array $[12,13]$. During the flight, the probe will rotate around its axis, and not be in a stationary state like a satellite. It is possible to assume that the solar array generation will be slightly higher than the generation in the case of illumination of one side, since at certain moments of time the spacecraft will turn to the sun with its edge and 2 sides will be illuminated, albeit not at an optimal angle $[14,15]$.

\section{Development and production of a solar array for the stratospheric apparatus}

To produce a solar array of $0.01 \mathrm{~m}^{2}, 16$ monocrystalline SPs of $390 \mathrm{~mm}^{2}$ each were required for each side of the CubeSat cube. In total, 2 cubes require $8 \mathrm{SAs}$ according to the number of sides. 
To create 1 solar array, one needs to combine 16 SPs into a sequential branch. For the consistent integration of SP into a single electrical circuit, there are some requirements and conditions that must be met and observed. When SPs are connected in series, an electrical branch is formed, through which only one current can flow, and the voltages generated by all SPs are added. Thus, the total voltage will be equal to the total one, and the total current will be limited by the SP with the lowest current output. The current output of the SP is influenced by:

- Rated voltage value.

- Solar panel size.

- Efficiency coefficient of SP.

- Serviceability of SP.

- Temperature of SP.

All SPs connected in series must have the same characteristics and be serviceable. The characteristics of SPs, even from the same batch, may differ. Some SPs in the batch may be defective or damaged in transit.

After receiving the ordered consignment of SPs, their visual inspection was carried out, unfortunately, about $20 \%$ of them turned out to be broken (Fig. 1.).

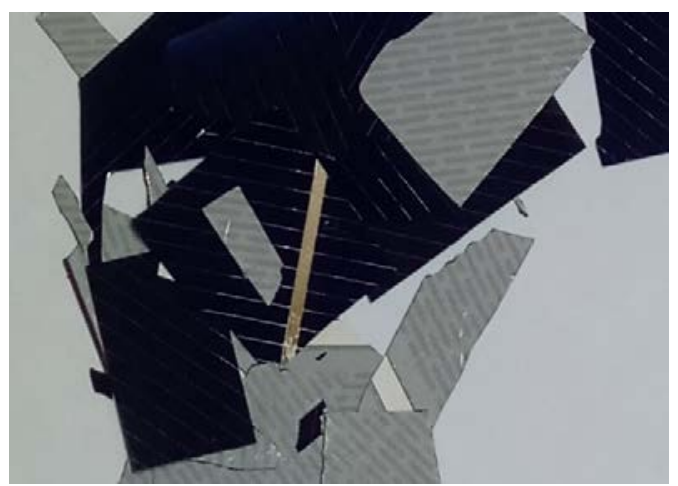

Fig. 1. Solar panels damaged in delivery

After sorting and removing faulty SPs from the received batch, the incoming control of the compliance of SP parameters with the manufacturer's declared parameters was carried out. For this purpose, the input control of SP parameters was carried out.

An experimental device was developed and assembled to carry out the input control of the SPs, check their serviceability and diagnose the output parameters (Fig. 2.). The device included a closed body, the walls of which were pasted over with a reflective material, and a lamp, which was fixed on the lid. The lamp was chosen to provide the required intensity of the luminous flux for the maximum power of the SP.

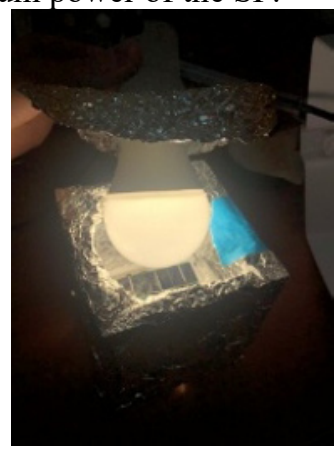

Fig. 2. Test stand for SP 
The parameters of the SP operation according to the manufacturer's data were $500 \mathrm{mV}$ and $125 \mathrm{~mA}$ or $130 \mathrm{~mA}$ in case of a short circuit. A SP with measuring devices connected to the terminals was installed in the device, after which the lid was closed, the light was turned on, and the parameters of the SP were recorded from the measuring devices. After the check, the SPs, the parameters of which significantly differed from those established by the manufacturer, were eliminated.

For clarity, the graph (Fig. 3.) shows the number of deviations of the SP parameters per 100 pieces. The graph shows that for 100 SPs - 24 pieces were found to have latent defects. Of these, 8 SPs did not give out the declared voltage, deviations in the declared short-circuit current had 9 SPs, 7 SPs had deviations in both current and voltage. Thus, out of 100 visually serviceable SPs, 24 SPs had characteristics that did not allow their use for the SP of the spacecraft. Unfortunately, it is impossible to draw a conclusion about the reasons for the discrepancy between the output parameters of the SP declared by the manufacturer. Possible reasons for this:

- Damage of SPs during transportation.

- $\quad$ Manufacturing defect.

- $\quad$ SPs are secondary after the processing of faulty (broken), large SPs.

- Rapid degradation of SPs after production.

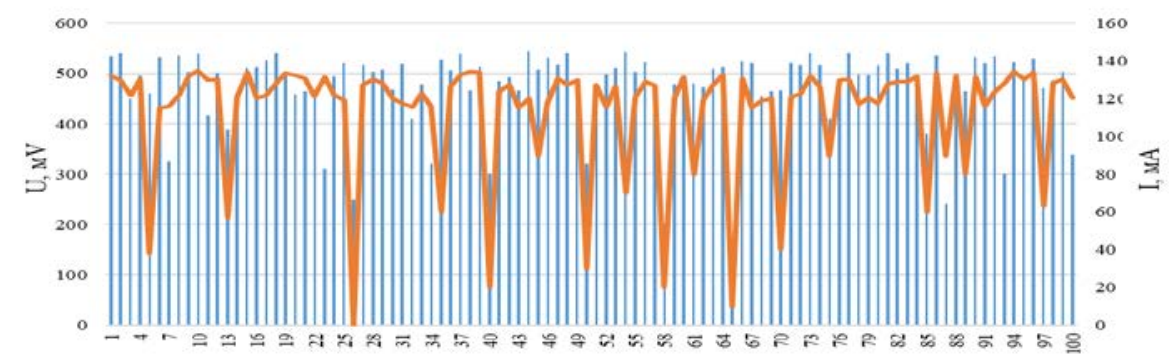

Fig. 3. The obtained parameters - current and voltage - of the SP's output after tests on the developed device

After sorting SPs from the serviceable samples, the SAs were mounted. The solar array consisted of 16 SPs connected in series, and had characteristics of about $8 \mathrm{~V}$ and $0.125 \mathrm{~mA}$ of the output current, with solar remediation of at least $1000 \mathrm{~W} / \mathrm{m}^{2}$. To determine the point of maximum power of all solar panels used on the module of the stratospheric apparatus (2 modules of 4 SAs each) and to adjust the MPPT controller, we tested them. The test results for finding the point of maximum power of the SA are shown in Fig. 4.

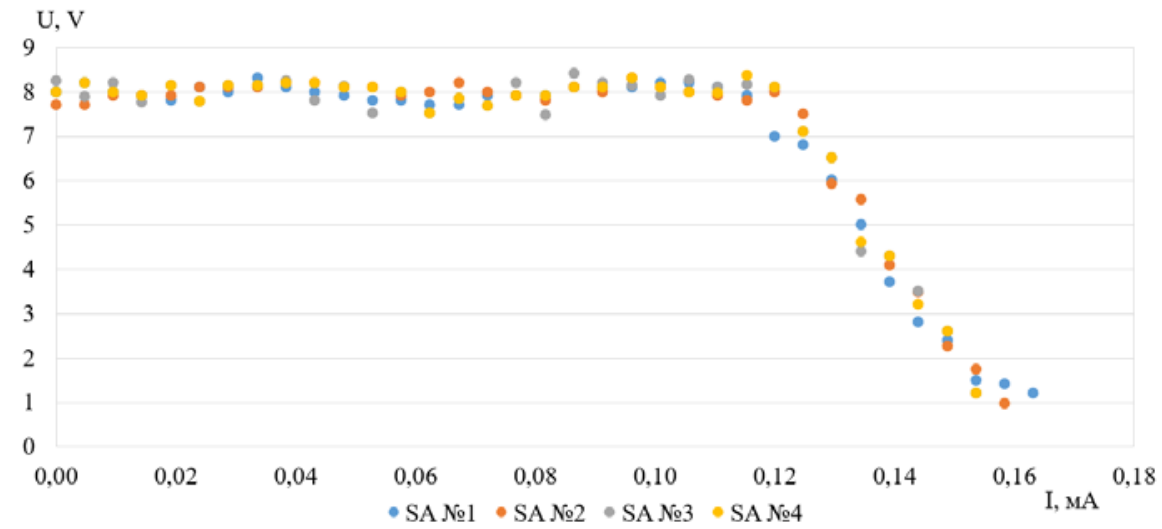

Fig. 4. Search for the point of maximum power of the solar array by the current-voltage characteristic 
The averaged point of maximum power for a set of 4 SAs is in the region of voltage 7.9 $\mathrm{V}$ and current $125 \mathrm{~mA}$. The current-voltage characteristic of the SA was used to create an MPPT controller that ensures the maximum efficiency of the SA for each CubeSat module.

The next step after the development and creation of the solar array was to work out how to mount it on the CubeSat $2 \mathrm{U}$. To calculate the parameters of strength and ergonomics of the SA attachment to the stratospheric apparatus, its 3D model was built with the SA installed (Fig. 5.).

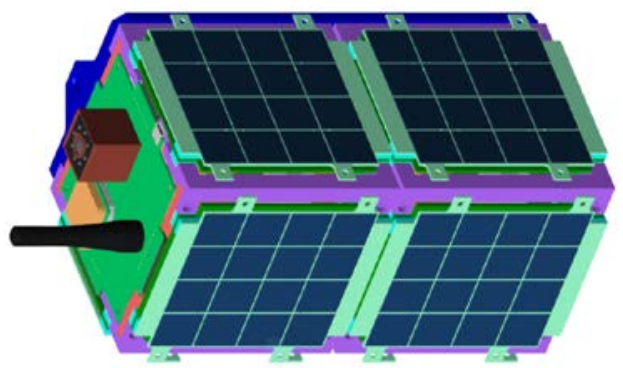

Fig. 5. 3D model of the stratospheric apparatus CubeSatU2 with installed SAs

The study of several ways of attaching the solar array to the surface of the probe has shown that the most optimal method of attaching the solar array is to fasten it with screws. To attach the solar array to the stratospheric apparatus, a special form-gasket was designed, then printed on a $3 \mathrm{D}$ printer (Fig. 6.).
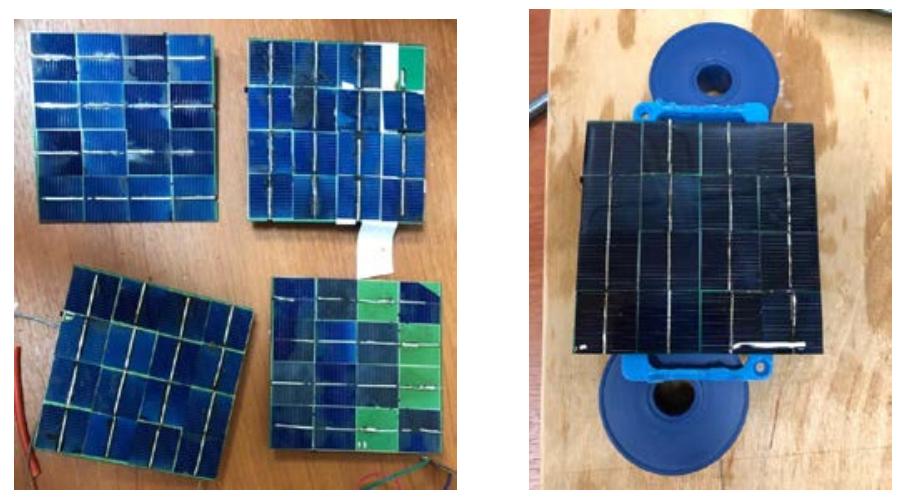

Fig. 6. SA for installation on a stratospheric apparatus

After installing the SA on the CubeSat $2 \mathrm{U}$, the assembled structure looked as shown in Fig. 7.

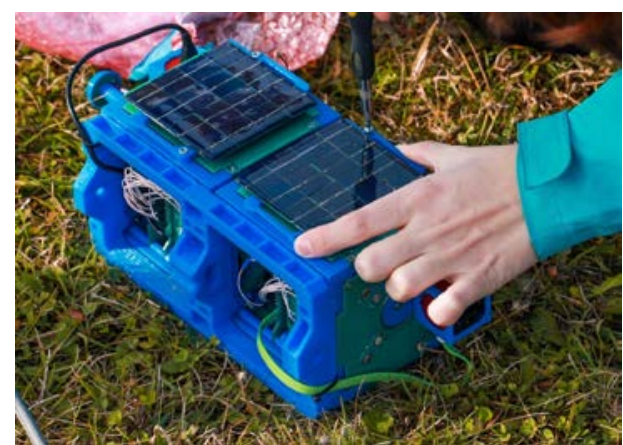

Fig. 7. CubeSat $2 \mathrm{U}$ with installed SAs 
The actual test of CubeSat $2 \mathrm{U}$ with the installed SAs on a stratospheric probe took place on October 5, 2020 at the Kameshkovo airfield, Vladimir region. The flight went on as usual and took two hours to ascend and about half an hour to descend. The photographs taken by the CubeSat $2 \mathrm{U}$ during the flight are shown in Fig. 8.

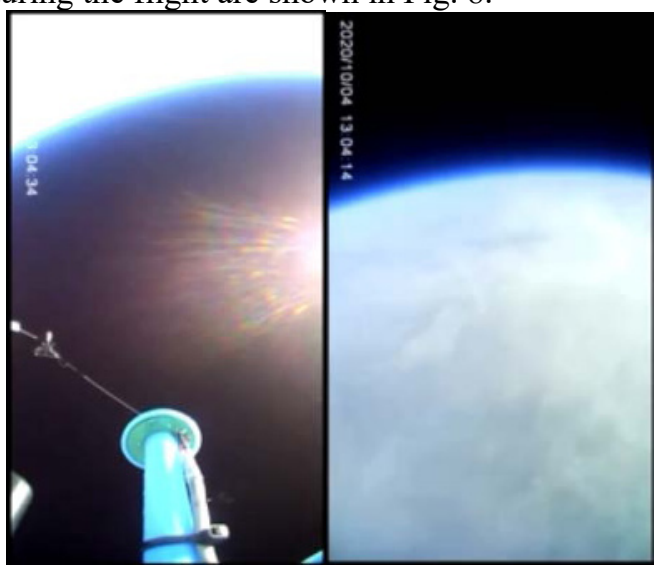

Fig. 8. Photos taken by CubeSat $2 \mathrm{U}$ space probe

After landing, the device was found $70 \mathrm{~km}$ from the launch site. The initial inspection did not reveal any serious mechanical damage to the solar array, except for the epoxy resin that had come off at the edges due to low temperatures. During the flight, the stratospheric vehicle measured the state of the atmosphere: pressure, temperature, humidity, concentration of gases and impurities, it obtained the flight path, generation of electricity from the SA and relative movements of the vehicle around the cable; as well as cameras installed on the top and bottom boards recorded the video of the flight.

\section{Results}

The volumes of electricity generation by the SA of the stratospheric apparatus CubeSat $2 \mathrm{U}$ during the flight, which lasted about 2.5 hours ( 2 hours of flight and 0.5 hours of landing), amounted to $5.165 \mathrm{~W}$. It is clear that during the flight in each unit of time one of the sides of the CubeSat $2 \mathrm{U}$ with solar panels was turned towards the sun; if the edge of the CubeSat $2 \mathrm{U}$ was turned towards the sun, then the sun's rays fell on two sides of the apparatus, but at rather sharp angles, which negatively affected the efficiency of the solar array. Let us recall that all the sensors and computing power of the CubeSat $2 \mathrm{U}$ consumed 10.718 watts during the flight. This value does not take into account the power of heating devices, since the issue of the need and intensity of heating of stratospheric devices still needs to be worked out, and in the process of working out the power consumed by heating elements will inevitably decrease due to optimization of the heat balance and the use of energy-saving technologies. Thus, in total, all the electronics of the CubeSat $2 \mathrm{U}$ consumed about $10.7 \mathrm{~W}$ during the flight, and the SA generated $5.1 \mathrm{~W}$, which is half of the power consumed. The generation of half of the power consumption of the SA of the stratospheric apparatus without using the final (reserve) fuel resources can be considered a good result. Improving energy efficiency, energy saving modes and developing optimal operating modes for electronics using sleep and interrupt modes for the CubeSat $2 \mathrm{U}$ will further reduce the amount of power consumed.

Separately, it should be noted that the stable power generation of the solar arrays was even present at critical subzero temperatures, which favorably distinguishes them from Liion batteries. 
The solar batteries cannot completely replace the Li-ion batteries due to general insufficient generation of electricity and dips in its generation, however, the solar arrays provide 'insurance' for the power supply in the event of a voltage drop on the Li-ion battery due to low temperatures, which does not allow the main electronic systems to be switched off, as well as the use of a solar array will further reduce the number of Li-ion batteries from 4 cells in each CubeSatU2 cube to 2 pieces, that is, by half, which is a good result. Since the SAs are not very heavy, the weight balance with the exclusion of Li-ion batteries and addition of SAs turns out to be negative, that is, an effect is obtained in reducing the weight of the device.

\section{Discussion}

The carried out scientific work gave the following results. The method of testing electronic equipment of spacecraft on stratospheric probes has proved to be very effective. This method enables one to check the developed and manufactured electronic equipment in practice in conditions similar to those in which it will be used, but at the same time has a much lower cost. The reliability of the developed electronic equipment and the solar array was $100 \%$.

The necessity of the input control of the SP for the production of the solar array has been proved. In the received batch of 100 SPs, 24 SPs were found to be defective and had unsatisfactory characteristics. The developed methodology for the input control of the SPs and diagnostics of the output characteristics produced by the solar array has shown its effectiveness and made it possible to ensure reliable operation of all solar arrays on the CubeSat2U.

\section{Conclusion}

The launch of the CubeSat2U with the SA on board showed that the generated volume of electricity by the SA during the flight was $5.1 \mathrm{~W}$. The generated electricity by the SA satisfies $50 \%$ of the consumption of electronic equipment in the stratospheric apparatus. The solar arrays cannot fully satisfy the needs of the stratospheric apparatus for electricity. The main reasons are insufficient volumes of power generation due to small surface area of the solar array and the lack of stability in generation - power failures. However, the use of SAs makes it possible to compensate for the dips in the current output of the Li-ion battery at moments of negative temperatures, as well as to reduce the amount of Li-ion battery on board the spacecraft, which saves weight. The resulting savings can be used to install additional equipment on the CubeSat type of spacecraft.

Some results were obtained within the framework of the scholarship program of the President of the Russian Federation for young scientists and postgraduate students No. SP-4607.2021.1.

\section{References}

1. D. Swanson, «The SunPower story», 2008 IEEE Hot Chips 20 Symposium (HCS), Stanford, CA, 2008, pp. 1-54, doi: 10.1109/HOTCHIPS.2008.7476556.

2. D. J. Flood, «Satellite solar energy systems», Proceedings of Power and Energy Systems in Converging Markets, Melbourne, Victoria, Australia, 1997, pp. 12-, doi: 10.1109/INTLEC.1997.645857. 
3. YERGIN, D. (2012). The quest: energy, security and the remaking of the modern world. NewYork, PenguinBooks.

4. Vlasov A., Yuldashev M. Performance Analysis of Algorithms for Energy-Efficient Data Transfer in Wireless Sensor Networks // 2019 International Conference on Industrial Engineering, Applications and Manufacturing, ICIEAM 2019. 2019. N. 8743087.

5. R.S. Joshi and S. B. Gupta, «Diagnostic of Neutralization Current for Arcs on Satellite Solar Panel Coupons», in IEEE Transactions on Plasma Science, vol. 43, no. 9, pp. 3000-3005, Sept. 2015, doi: 10.1109/TPS.2015.2456212.

6. V. Golovko, A. Kroshchanka, S. Bezobrazov, A. Sachenko, M. Komar and O. Novosad, «Development of Solar Panels Detector», 2018 International ScientificPractical Conference Problems of Infocommunications. Science and Technology (PIC S\&T), Kharkiv, Ukraine, 2018, pp. 761-764, doi: 10.1109/INFOCOMMST.2018.8632132.

7. T. A. Cross, R. Kimber and C. Goodbody, «Solar panels for microsatellites: GaAs, GaAs/Ge and beyond», Proceedings of 1994 IEEE 1st World Conference on Photovoltaic Energy Conversion - WCPEC (A Joint Conference of PVSC, PVSEC and PSEC), Waikoloa, HI, 1994, pp. 1970-1977 vol.2, doi: 10.1109/WCPEC.1994.520754.

8. R. Aslan et al., «Space Technology Capacity Building in Support of SDG 2030 Through CubeSat SharjahSat-1», 2019 9th International Conference on Recent Advances in Space Technologies (RAST), Istanbul, Turkey, 2019, pp. 955-958, doi: 10.1109/RAST.2019.8767863

9. K V Selivanov, A I Vlasov and V A Shakhnov Analysis of factors affecting the capacity of LI-ION rechargeable batteries at low temperatures // Journal of Physics: Conference Series. Conference on Applied Physics, Information Technologies and Engineering. (APITECH-II-2020). V.1679. N022053.

10. Vlasov A.I., Demin A.A. Visual Methods of Formalization of Knowledge in the Conditions of the Synchronous Technologies of System Engineering // ACM International Conference Proceeding Series. 2017. N. 3166098.

11. Prudius A.A., Karpunin A.A., Vlasov A.I. Analysis of machine learning methods to improve efficiency of big data processing in Industry 4.0 .. Journal of Physics: Conference Series. 2019. N. 032065.

12. Selivanov, K \& Vasiliev, I \&Lyuminarskaja, E. (2020). Microcontroller-based electromechanical drive. IOP Conference Series: Materials Science and Engineer-ing. 709. 022024. 10.1088/1757-899X/709/2/022024.

13. Garifulina MR, Vlasov AI, Makarchuk VV, Adamovik N. Model of a solar battery element of the CIGS type // Engineering Bulletin. 2012. No. 8. -12 p.

14. Selivanov K.V., Vlasov A.I., Shakhnov V.A. Analysis of factors affecting the capacity of li-ion rechargeable batteries at low temperatures // Journal of Physics: Conference Series. 2020. N. 22053.

15. Yuldashev M.N., Vlasov A.I., Novikov A.N. Energy-efficient algorithm for classification of states of wireless sensor network using machine learning methods // Journal of Physics: Conference Series . 2018. V. 1015. N. 032153. 